\title{
Effectiveness of Kinesio taping application on low back pain in university employees: A randomized controlled trial
}

\author{
Abdelrazek Youssef Ibrahim Bayomi \\ Department of Rehabilitation, Prince Sattam bin Abdul-Aziz University, Alkharj, Saudi Arabia.
}

Accepted 5 June, 2017

\begin{abstract}
The aim of this study was to investigate the therapeutic effect of Kinesio-taping on treatment of low back pain. Twenty male university employees (with low back pain) aged 30 to $40 \mathrm{yrs}$, were randomly classified into two groups: Kinesio taping group (KT) comprised of ten patients, who obtained Kinesio taping application in addition to home exercise for back and abdominal muscles; and control group which comprised of ten patients, who received only home exercise recommendations without KT application. Pain and disability scores were recorded before starting the program and after 6 weeks at the end of the program. Baseline characteristics showed non-significant differences between the two groups $(p>0.05)$. There was significant difference between pre- and post-treatment in the KT group $(p<0.05)$; while the control group showed non-significant differences between pre- and post-program ( $p>0.05)$. It could be concluded that KT is a beneficial adjunctive physical therapy tool that could help in decreasing disability and pain.
\end{abstract}

Keywords: Low back pain, Kinesio taping, university employees.

E-mail: dr.razekk@gmail.com. Tel: +966540435859.

\section{INTRODUCTION}

Low back pain (LBP) is a very common health problem worldwide and it is a cause of disability and has negative effect on performance at work and general well-being. LBP can be acute, sub-acute, or chronic (Katz, 2006). LBP was identified as the most common cause of disability in young adults, with more than 100 million workdays lost per year. Previous survey suggested that LBP accounted for a quadrupling of the number of work days lost from 7 million in 1980 to 28 million by 1987 (Croft et al., 1993).

The main symptom of LBP is the pain or the ache in the lower back and sometimes it comes down to the buttocks and legs in the common cases clearly within a short period, muscle weakness, decreased activity, and even impairment in body functions is consequent to pain problem. LBP has many risk factors such as pregnancy, strenuous physical work, overweight (Nordqvist, 2016; Engers et al., 2003). Some problems of the back as a strain of muscle or ligament, muscle spasm, improper lifting, structural problems, such as bulging disk, sciatic nerve pressure, abnormal curvature of the spine such as scoliosis and bad sleep postures will cause LBP (Roberts et al., 2006).

The 2010 Global Burden of Disease Study (Lopez and Murray, 1998) estimated that LBP is among the top 10 diseases and injuries that account for the highest number of DALYs worldwide. Prevalence of LBP among adults is about 70 to $80 \%$; in the general population there is at least one episode of LBP at some time in their lives (Waker et al., 2004). The commonly prescribed treatment options are conservative and stretching, taping, laser therapy and drug therapy, hydrotherapy, electrotherapy and topical application. They have all been investigated and have shown variable clinical benefit (Smeets, 2009).

Kinesio-taping (KT) is a technique that has been used in the clinical management of people with chronic back pain; it was developed by Kenso Kase in the 1970s (Kase et al., 1996). He developed the tape over 40 years ago in Japan and introduced it to the United States in the 1990s (Kase et al., 2003). Therapeutic KT can benefit a wide 
variety of musculoskeletal and sports injuries, plus inflammatory conditions. It can be stretched to 120-140\% of its original length and conventional tape (Paoloni et al., 2011). It has a beneficial effect for normalization of muscular function, improvement of lymphatic and vascular flow, decrease in pain, and contributes to correcting possible joint misalignments (Thelen et al., 2008; Paoloni et al., 2011).

\section{Objectives}

The aim of this study was to investigate the therapeutic effect of Kinesio-taping on treatment of low back pain in university employees.

\section{MATERIALS AND METHODS}

\section{Subjects}

Twenty male university employees who were suffering from low back pain were selected for study from Prince Sattam Bin AbdulAziz University, Al Kharj, KSA. This study aimed to investigate the effect of 6 weeks application of KT on LBP. The participants were enrolled in this study, according to the following criteria; the participated subjects have LBP, their age ranged from 30 to 40 years. The participants were randomly classified into two groups; KT group comprised of ten patients, received Kinesio taping application in addition to home exercise for back and abdominal muscles and control group comprised of ten patients, received only home exercise recommendations without KT application. The patients who were suffering have undergone back surgery, neurological problem, cardiovascular disease, skin problems such as sensitive skin and wound, respiratory disease, and orthopedic problems were excluded from the study.

\section{Materials}

\section{For evaluation}

All subjects were evaluated before and after 6 weeks of KT application of the following materials:

a) Visual analogue scale (VAS): It is a simple and frequently used material for the assessment of variations in the intensity of pain. In clinical practice, the percentage of pain relief, assessed by VAS, is often considered as a measure of the efficacy of treatment. However, from the patient's perspective, this spectrum appears continuous their pain does not take discrete jumps, as a categorization of none, mild, moderate and severe would suggest. Operationally, a VAS is usually a horizontal line, $10 \mathrm{~cm}$ in length, anchored by word descriptors at each end (Lee and Yoo, 2012).

b) Oswestry low back pain disability questionnaire: The survey appeared overleaf is partitioned into ten areas chose from a progression of trial questionnaires intended to evaluate confinement of different activities of daily living (Wewers and Lowe, 2000).

\section{For treatment}

Kinesio tape (KT): The Kinesio taping technique was developed by Dr. Kenzo Kase in Japan more than 25 years ago. It is a thin, cotton, porous fabric with acrylic sticky. It has been theorized to improve a variety of physiological problems, including the range of motion and healing, based on the functions of the tape (Bennatt and Ritchie, 2001).

\section{Procedures}

\section{Evaluation}

a) Visual analogue scale: The patients were asked to mark on the line the point that they feel it represents their perception of their current state (e.g., "No pain" to "Unbearable pain"). The VAS score is determined by measuring in centimeter from the left hand end of the line to the point that the patient marks (Oliveria, 1999).

b) Oswestry low back pain disability questionnaire: This scale is used to describe patient's limits in action of daily functional activity caused by back pain. This was found to be the most suitable number for obtaining accurate assessments, without confusing the patient. It consists of 10 sections, each section has 6 statements. Every section is scored on a 0 to 5,5 representing the greatest disability. The scores for all sections are added together; giving a possible score of 50 . The total is doubled and expressed as a percentage. Interpretation of disability scores: 0 to $20 \%$ minimal disability the patient can adapt to most living exercises. Normally, no treatment is shown separated from counsel on lifting, sitting and work out. 21 to $40 \%$ moderate disability the patient encounters more agony and trouble with sitting, lifting and standing. Travel and social life are more troublesome and they might be crippled from work. Individual care, sexual movement and resting are not horribly influenced and the patient can for the most part be overseen by the moderate means. 41 to $60 \%$ sever disability, pain remains the principle issue in this gathering, and however exercises of daily living are influenced. These patients require detailed examination. 61 to $80 \%$ crippled back pain impinges on all parts of the patient's life. Positive intercession is required. 81 to $100 \%$ these patients are either bed-bound or overstating their indications.if patient marks two statements; the highest scoring statement is recorded as a true indication of his disability. If a section is not completed because it is inapplicable (e.g. Section 8sex life), the final score is adjusted to obtain a percentage (Pijinappel, 2007).

\section{Treatment}

Kinesio tape (KT): Before applying KT, the skin should be prepared first to prevent wrinkles and any errors. The area to be treated should be cleaned, free of hair to avoid sticking of hair when $\mathrm{KT}$ is removed. Taping should last for 3 to 5 days. After this period, KT can be easily removed through showering or making it wet.

The two (I) shapes of KT were applied from the origin of the lumbar erector spinae (Iliocostalis Lumborum) to its insertion. In the case of flexion disturbances, the patient was able to support herself during flexion. The first 4 to $5 \mathrm{~cm}$ of tape was carefully removed from its paper backing. The base of the tape was applied to the sacrum in the neutral position. The patient was asked to do a maximum flexion of the spine and the paper backing of the tape was removed, except for the final 4 to $5 \mathrm{~cm}$ and the tape were used on one side paravertebral in the direction of the cranium, under slight traction. Finally, the last 4 to $5 \mathrm{~cm}$ of the tape were applied without traction. The same procedure was then applied to the other side. The tape was scrubbed by hand several times to warm the adhesive material to achieve better adhesion (Fahad et al., 2013).

\section{Statistical analysis}

Descriptive statistics (mean and standard deviation) were computed 
for all outcome measures. Paired and unpaired t-tests were used to compare the mean differences of the study variables within and between both groups respectively. Level of significance was set at $p<0.05$ for all statistical tests. Statistical analysis was completed using SPSS, version 16.

\section{RESULTS}

Twenty male university employees suffering from low back pain were selected for study from Prince Sattam Bin Abdul-Aziz University. Their age ranged from 30 to 40 years old. Pain (VAS) and disability were recorded before starting the program and after 6 weeks at the end of the program. They evaluated every week for six weeks. The results were expressed as mean $\pm S D$, and $P$ values less than 0.05 were considered significant.
Baseline characteristics, there were no significant differences between the measured variables in the two groups as demonstrated in Table 1.

After 6 weeks of intervention, there were statistical significant differences between the two groups. In KT group, the pain (VAS) level was $6.8 \pm 0.83$, and disability level in Oswestry low back pain disability questionnaire showed $16.6 \pm 4.21$ before treatment and become (VAS) level $3.8 \pm 0.83$ and disability level became $7.8 \pm 0.83$ after treatment respectively. There were significant differences between pre- and post-treatment in the KT group. While in control group, VAS changed from $6.5 \pm$ 0.91 to $5.9 \pm 0.72$ and disability questionnaire changed from $15.9 \pm 3.88$ to $13.7 \pm 3.6$ at the end of the study with non-significant differences between pre- and postprogram as demonstrated in Table 2.

Table 1. Baseline characteristics of KT and control groups.

\begin{tabular}{lcccc}
\hline Characteristic & KT group $(\mathbf{n}=\mathbf{2 0})$ & Control group $(\mathbf{n}=\mathbf{2 0})$ & $\mathbf{P}$ - value & Significance $\mathbf{p}<\mathbf{0 . 0 5}$ \\
\hline Age (year) & $30.2 \pm 2.14$ & $31.4 \pm 1.7$ & 0.1819 & NS \\
Pain (VAS) & $6.8 \pm 0.83$ & $6.5 \pm 0.91$ & 0.4511 & NS \\
Disability & $16.6 \pm 4.21$ & $15.9 \pm 3.88$ & 0.7036 & NS \\
\hline
\end{tabular}

KT: Kinesio taping, Sig: significant, NS: non-significant.

Table 2. Statistical analysis of mean differences between the two groups at the end of the program.

\begin{tabular}{lcccccc}
\hline \multirow{2}{*}{ Variables } & \multicolumn{2}{c}{ KT group } & & \multicolumn{2}{c}{ Control group } & \multirow{2}{*}{ Post-treatment p-value } \\
\cline { 2 - 3 } \cline { 5 - 6 } & Pre-treatment & Post-treatment & & Pre-treatment & Post-treatment & \\
\hline Disability & $16.6 \pm 4.21$ & $2.8 \pm 0.83^{\mathrm{s}}$ & & $15.9 \pm 3.88$ & $13.7 \pm 3.6^{\mathrm{ns}}$ & $<0.0001^{\mathrm{s}}$ \\
VAS & $6.8 \pm 0.83$ & $3.8 \pm 0.83^{\mathrm{s}}$ & & $6.5 \pm 0.91$ & $5.9 \pm 0.72^{\mathrm{ns}}$ & $<0.0001^{\mathrm{s}}$ \\
\hline
\end{tabular}

KT: Kinesio taping, VAS: visual analogue scale, S: significant, NS: non-significant.

\section{DISCUSSION}

The aim of the present study was to investigate the effects of KT in the treatment of LBP in university employees. The KT was applied to patients with low back pain. In this study, significantly greater reductions in disability and pain intensity were obtained earlier in patients who have low back pain who received Kinesio taping.

Although the mechanism through which KT acts on musculoskeletal conditions is not yet clear, it is supposed to KT applies pressure to the skin or lengthens the skin and that this external load may stimulate cutaneous mechanoreceptors (large myelinated fibers) and inhibit pain transmission according to the gate control theory (Braggins, 2000).

Melzack and Wall proposed the gate control theory, which subtracts that the spinal cord contains a neurological 'gate' that either blocks pain signals or allows them to continue on to the brain (Melzack and Wall, 1965). It is also supposed that the skin will be raised due to the flexibility of the tape, creating a wider space between the skin and the muscle, leading to elaboration of blood circulation and drainage of lymphatic fluids in the taped area, that way decreasing pain, increasing ROM and mending ADL. Previous studies have suggested that KT may enhance proprioceptive afferent feedback (Pijinappel, 2007; Gill and Callaghan, 1998).

The result of this study comes with agreement the study done by Paoloni et al. (2011), which observed a highly significant reduction in disability when using $\mathrm{KT}$ in the study group. This reduction in disability could be referred to the younger age subjects of the KT group (30 to 40 years) in the present study, when compared to the age group of the subjects (62 years) taken by Paoloni et 
al. (2011) study investigated the effect of a combination of exercise and KT on pain and ADL in patients with LBP. Our findings in terms of reducing LBP were consistent with the results of Paoloni et al. (2011), who observed a highly significant reduction in pain, measured using a VAS, after four weeks of treatment with KT.

Also, Castro-Sánchez et al. (2012) compared the effectiveness of Kinesio taping and sham Kinesio taping in patients with chronic low back pain. They reported significant short-term improvements in pain intensity in the Kinesio taping group (Castro-Sánchez et al., 2012). Merino et al. (2010) found that hip and lower back flexibility have a significant increase in sit-bend distance after KT applied. Also, González-Iglesias et al. (2009) assessed cervical range of motion before and after taping and revealed that all directions of the cervical spine movement had significantly improved. These studies also applied KT from insertion to the origin, direction (González-Iglesias et al., 2009).

Another study tested the effects of a single application of Kinesio taping compared with Micropore (placebo group) taping and a control group with no intervention in patients with chronic low back pain for the results of pain intensity and disability. This is the first study that compared the Kinesio taping method with Micropore taping as a form of placebo therapy. They observed that, although the Kinesio Taping group showed an improved disability score. All other statistical comparisons between groups showed no statistical significance. These findings raise a question regarding the use of Kinesio Taping in clinical practice for patients with chronic low back pain from the effects observed (small) appears (Maurício et al., 2015).

Some studies come with disagreement with the present results as a study done by McConnell et al. (2011) who showed no significant effect of shoulder taping on maximum shoulder abduction ROM and total reduction of pain. Also, Akbas et al. (2011) approved that there is no effect of KT on pain in patients with Patellofemoral Pain syndrome.

However, more studies used to measure the effectiveness of KT and there was some difference with the present study because some of these studies find no beneficial effect of KT, the present study also finds more studies proved that there is a more beneficial effect for $\mathrm{KT}$. So, the findings of this study continue to search to know if $K T$.

\section{Conclusion}

It has been concluded that Kinesio taping has significantly greater reductions in disability and pain intensity in patients who have low back pain. It could be concluded that KT is a beneficial adjunctive physical therapy tool that could help in decreasing disability and pain.

\section{ACKNOWLEDGEMENT}

The author would like to thank all subjects who participated in this study.

\section{REFERENCES}

Akbas E, Atay AO, Yuksel I, 2011. The effects of additional Kinesio taping over exercise in treatment patellofemoral pain syndrome. Acta Orthop Trumatol Turc, 45(5): 335-341.

Bennatt AE, Ritchie R, 2001. Questionnaires in medicine: a guide to their design and use. Oxford university press.

Braggins S, 2000. Treatment and prevention of low back pain. Back Care: A Clinical Approach. London: Harcourt Publishers Limited, pp: 137-8.

Castro-Sánchez AM, Lara-Palomo IC, Matarán-Peñarrocha GA, 2012. Fernández-Sánchez M, Sánchez-Labraca N, Arroyo-Morales $M$ Kinesio Taping reduces disability and pain slightly in chronic nonspecific low back pain: a randomised trial. J Physiother; 58(2): 89-95.

Croft P, Rigby AS, Boswell R, Schollum J, Silman A, 1993. The prevalence and characteristics of chronic widespread pain in the general population. J Rheumatol, 20: 710-713.

Engers $A$, Jellema $P$, Wensing $M$, van der Windt $D$, Grol $R$, van Tulder MW, 2003. Individual patient education for low back pain. Cochrane Database Syst Rev, 3: 20-60.

Fahad A, Ashraf R, Abdul Rahim Z, Abdulaziz A, Syamala B , Ganeswara R, 2013. Kinesio taping for the treatment of mechanical low back pain. World Appl Sci J, 22(1): 78-84.

Gill KP, Callaghan MJ, 1998. The measurement of lumbar proprioception in individuals with and without low back pain. Spine, 23: 371-377.

González-Iglesias J, Fernández-de-Las-Peñas C, Cleland JA, Huijbregts P, Del Rosario Gutiérrez-Vega M, 2009. Short-term effects of cervical Kinesio Taping on pain and cervical range of motion in patients with acute whiplash injury: A randomized clinical trial. $J$ Orthopaed Sports Phys Ther, 39(7): 515-521.

Kase K, Tatsuyuki H, Tomoko O, 1996. Development of kinesio tape. Kinesio taping perfect manual. Kinesio Taping Association, 6: 117118.

Kase K, Wallis J, Kase T, 2003. Clinical Therapeutic Applications of the Kinesio Taping Method. Tokyo, Japan: Ken Ikai Co Ltd. pp: 40-55.

Katz JN, 2006. Lumbar disc disorders and low-back pain: socioeconomic factors and consequences. J Bone Joint Surg Am, 88(2): 21-24.

Lee JH, Yoo WG, 2012. Application of posterior pelvic tilt taping for the treatment of chronic low back pain with sacroiliac joint dysfunction and increased sacral horizontal angle. Phys Ther Sport, 13: 279-285.

Lopez AD, Murray CC, 1998. The global burden of disease, 19902020. Nat Med, 4(11): 1241-1243.

Maurício ALJ, Sousa MV, Neves LA, Cezar AA, Costa LO, 2015. Kinesio Taping is not better than placebo in reducing pain and disability in patients with chronic non-specific low back pain: a randomized controlled trial. Braz J Phys Ther, 19(6): 482-490.

McConnell J, Donnelly C, Hamner S, Dunne J, Besier T, 2011. Effect of shoulder taping on maximum shoulder external and internal rotation range in uninjured and previously injured overhead athletes during a seated throw. J Orthop Res, 29(9): 1406-1411.

Melzack R, Wall PD, 1965. Pain mechanisms: A new theory. Science, 150: $971-978$.

Merino R, Mayorga D, Fernández E, Torres-Luque G, 2010. Effect of Kinesio taping on hip and lower trunk range of motion in triathletes: $A$ pilot study. J Sport Health Res, 2(2): 109-118.

Nordqvist C, 2016. Back pain: causes, symptom and treatments. Medical news today, 2016: 1 .

Oliveria $R, 1999$. Soft tissue injuries in sports people: The contribution of Kinesio taping. 15th Annual Kinesio Taping International Symposium Review, 6: 13-23.

Paoloni M, Bernetti A, Fratocchi G, Mangone M, Parrinello L, Del Pilar Cooper L, Sesto L, Di Sante L, Santilli V, 2011. Kinesio taping 
applied to lumbar muscles influences clinical and electromyographic characteristics in chronic low back pain patients. Eur J Phys Rehabil Med, 47(2): 237-244.

Pijinappel H, 2007. Handbook of medical taping concept. 1. Madrid: Aneid Press.

Roberts S, Evans H, Trivedi J, Menage J, 2006. History and pathology of the human intervertebral disc. J Bone Joint Surg Am, 88(2): 10-14.

Smeets RJ, 2009. Do lumbar stabilising exercises reduce pain and disability in patients with recurrent low back pain? Austral $\mathrm{J}$ Physiother, 55: 138-200.

Thelen MD, Dauber JA, Stoneman PD, 2008. The clinical efficacy of kinesio tape for shoulder pain: a randomized, double blinded, clinical trial. J Orthop Sports Phys Ther, 38(7): 389-395.

Waker BF, Muller R, Grant WD, 2004. Low back pain in Australian adults: prevalence and associated disability. J Manipulat Physiol Therapeut, 27: 238-244.

Wewers ME, Lowe NK, 2000. A critical review of visual analogue scales in the measurement of clinical phenomena. Res Nurs Health, 13(4): $227-236$.
Citation: Bayomi AYl, 2017. Effectiveness of Kinesio taping application on low back pain in university employees: A randomized controlled trial. Int Res J Med Med Sci, 5(2): 25-29. 